\title{
A Study on Publicity and Its Realization in Public Art Design
}

\author{
Liping Liu ${ }^{1, a}$ \\ ${ }^{1}$ Tianjin Polytechnic University, Tianjin City, 300387, China
}

Keywords: Public Art Design; Publicity; Realization Strategy

Abstract. With the development of the society and the improvement of economic level in China, people have proposed higher demands on spiritual life while they get satisfaction in material life. In this context, the relationship between art and the public is getting closer and closer. Public art design aims at making people's living environment more artistic. Therefore, public art design has become a hot topic in the art research, and people pay more and more attention to the realization of publicity in public art design. Based on the author's learning and practical experience, this paper first analyzed the embodiment of publicity in public art design, and then put forward its realization strategies.

\section{Introduction}

Public art design is a hot topic in the field of architecture and design. On one hand, public art design practice is carried out in full swing. On the other hand, comments and discussions on public art design become a popular topic in design and the theoretical cycle. Its background is the rapid development of China's urbanization. It is thus clear that public art design which is an ancient and young discipline, has been integrated into the social and economic development. It is a great fortune in the design field. The purpose of this paper is to initiate the designer's deeper thinking about public art design.

\section{The Embodiment of Publicity in Public Art Design}

Public art design is subject to the inherent constraints of the public environment. First, the emergence of public art design is the results that art or artworks extend from artists' personal space to the public space. Art works in art galleries and other places have been open to the public environment, which is not just a simple displacement of space, but also a change in the inherent characteristics of art works. That is the emphasis on publicity in the art and the increase of environmental factors' constraints on the art. Public art design has been extended by the public environment.

Second, art design is subject to the public environment. The biggest difference between public art design and pure art works or the easel art works is the difference in space constraints and the latter is almost free from the impact of the spatial environment. For example, Van Gogh's masterpiece "sunflower" will not be affected its charm as the world famous painting style wherever it is displayed; the former is constrained by the public environment all the time. A public art design that can not be integrated into the public environment is always an unqualified work.

Third, public environments with different functions put forward different requirements on the public art design. Therefore, we can not evaluate these works with the same standard. For example, in the commemorative, symbolic and grand public places, the public art works should be solemn, majestic and massive. Public art design is subject to the public environment. Every public 
environment gets the kind of public art design with appropriate form, function and the purport; public environmental space gives birth to public art design and affects the artistic effect of public art design. It is the first shell of public art design and an inseparable part of the design. In addition, the view in evaluating a public art design works is different from assessing a pure art or easel artwork.

Public art design should reflect the common will of the public. First, the main body of public art design investment is the public. The input of public art design is both the behavior of the government or the social public welfare organization, but also the behavior of the individual or the enterprise. However, it is mainly the public behavior of the government, because the latter behavior is managed by the government. In this sense, public art design investment is a typical public behavior. The public is often identified as taxpayers and they invest public art design directly or indirectly. No matter what the way is, the main body of public art design investment is the public undoubtedly.

Second, the decision-making power of public art design works is in the hands of the public. Public art design is not an individual act by an artist or a designer. The creation or design rights belong to the artist, but whether it is adopted depends on the willingness and recognition of the public. Therefore, countries or regions with excellent public art design in the world have a complete set of systems and methods to ensure the objective, fair and impartial exhibition of public art design works.

Third, rights of use and judgment on public art design works are controlled by the public. In a broad sense, public art design is designed for the public, whose starting and end point are the public, so rights of use and judgment on public art design works is in the hands of the public. The relationship between public art and the public is not only interactive, but also constrained mutually. The mass and public society put forward the requirement on publicity of public art. Moreover, public art design also puts forward requirements on public spirit, public consciousness and public morality of the mass and public society.

Public art design should embody the artistic taste of the public. First, the public art design should reflect the mainstream artistic taste during a certain period. Public art design should be aesthetic in the first place and most people think that it is beautiful. During a certain period, people's aesthetic thought is brilliant with hierarchy. There always exists a relatively mainstream and dominant aesthetic style, which should become the main aesthetic keynote for the public. Public art design takes its responsibility for transforming and purifying people's good minds. If it only caters to people's likes and meet the vulgar taste of some people, it will degrade social conduct and public cultural taste in the name of public art, which is also contrary to the fundamental purpose of public art.

Second, public art design should fully reflect the geographical characteristics of the public environment. The public environment of public art design is always open public space with distinctive geographical characteristics, history and culture. Public art design is the carrier of regional characteristics, history and culture. The limited material form carries unlimited spiritual content. Excellent public art design works integrate the form of art, local characteristics and historical context ingeniously. Moreover, public art design works with history and culture will have a subtle influence on people. 
Third, the public art design should convey the excellent, timeless and traditional ethics. As a human art design of living, public art design also has a certain social function. Obviously, different times have different social ethical norms, different ways to carry forward them and different realization ways. As an important part of the public living space, public art design is not the main tool for advocating the mainstream social ethics, but it play an obvious role in shaping people's mind, cultivating people's will and influencing people's sentiment.

\section{The Realization Ways of Publicity in Public Art Design}

To respect the will of the public. First, public art should respect the participant role of the public. Under general circumstances, the investor of public art design is the government or social group, so the public design art investment is a public behavior. The public are taxpayers, so they are investors of public art design. In other words, the public indirect investors in public art design, so the public art designers need to communicate with the public and create the art works based on communication, so as to meet the public requirement on public art design and achieve the publicity in art design public.

Second, public art design should respect the public's decision-making power, use right and judgment right on the public art. The meaning of publicity in public art is not only reflected in that the public art involves the public to watch or appreciate, but also reflected in that the creation process of public art design expresses the public opinion. Since the service object of public art design is the public, only the use and judgment of the public on public art can evaluate and determine the artistic quality. Therefore, the public should have rights to use, judge and criticize the public art design. Public art design should not only try to meet the public demand, and but also should accept the appreciation and evaluation of the public after it is completed. Furthermore, designers should be aware of that to improve the public art design evaluation mechanism and to make this mechanism mature is an important force to promote the development of public art design.

To coordinate with aesthetic taste of the public and the overall environment. The development of public art design is not sound in China, which mainly manifests the problems of copy, comparisons or blind quantification of public art design in many cities. In the construction of public art design, there lacks consideration of urban environment, nature, humanities and development situation, which wastes a lot of creative resources and cannot meet the public's aesthetic taste. Moreover, it degrades the art taste of the city. The publicity of public art design is a reflection of the public attention, participation and recognition of art, so public art design should be democratic, advanced and open. In order to make beauty-appreciation of public art design close to the mass, it must suit both refined and popular tastes. The public art can meet the public aesthetic needs by the virtue of the coordination between the culture and environment in a specific area and the understanding of people's aesthetic taste and concern.

Public art design should convey public spirit. First, public art design should have the theme of peaceful development of human society. Peace and development are themes of the present era, and they are themes that public art design should attach importance to. Some negative themes are contrary to the publicity of public art design, such as war, violence and terror. Therefore, public art designers should recognize their own responsibility. It is very important to realize the publicity of public art 
design by expressing the theme that can optimize people's spiritual life and attaching importance to the social function of public art design to promote the development of public art design.

Second, public art design should show common and democratic humanistic care. To make art into the public space is the basis for the realization of publicity in public art design, so that democracy and popularization of the public art design is the fundamental way to promote the realization of publicity. In the process, designers should make art works attractive to the public. Popularization and democratization of public art design is to make the public experience beauty that public art design endows public space, so that the public can have a more pleasant mood by tasting connotation and details of art design. Therefore, when the public experience the humanistic care, they can approach art, and really fall love in art.

\section{References}

[1] Wang Haiyan. A Research of the Public Art Involved in Urban Public Space Design [J]. Art Science and Technology, 2014, (12): 20.

[2] Cao Ruilin, Sun Le. An Analysis of Public Space Design Psychology [J]. Popular Arts, 2014, (18): 82-83.

[3] Wen Rongdan. A Discussion on the Realization of Publicity in Public Art [J]. Modern Decoration (Theory), 2013, (08): 119-120.

[4] Wang Jue. An Imprint of Public Art [J]. Art Science and Technology, 2013, (01): 85.

[5] Ji Xiang. Architecture: Public Art [J]. Chinese and Overseas Architecture, 2012, (08): 1.

[6] Liu Yue. A Study on Design Ethics and the Development of Public Art Design in China [J]. Modern Decoration (Theory), 2011, (07): 41-42.

[7] Sun Jinde. A Discussion on Sculpture Design in Public Art [J]. Art Journal, 2012, (01): 45-47. 